\title{
The Role of Individualized Exercise Prescription in Type 2 Diabetes Mellitus Management
}

\author{
John Doupis, Konstantinos Karras, Konstantinos Avramidis \\ Department of Internal Medicine and Diabetes, Salamis Naval and Veterans Hospital, Salamis Naval Base, Attiki, Greece
}

DOI: https://doi.org/10.17925/EE.2021.17.1.2

$\mathrm{T}$ he adoption and the maintenance of a proper training routine are critical elements for optimal blood glucose management and overall health improvement in individuals with diabetes. Physical activity reduces cardiovascular risk, contributes to weight loss and improves overall wellbeing. A combination of aerobic and resistance exercise maximizes the benefit of daily training. The risk of exerciseinduced complications varies depending on the activity type and the presence of diabetes-related complications. Prescribing a personalized exercise programme may reduce the risk and maximize the benefit of physical activity in patients with diabetes.

\section{Keywords}

Diabetes, exercise, physical activity

Disclosures: John Doupis, Konstantinos Karras and Konstantinos Avramidis have nothing to disclose in relation to this article.

Review Process: Double-blind peer review.

Compliance with Ethics: This study involves a review of the literature and did not involve any studies with human or animal subjects performed by any of the authors.

Authorship: The named authors meet the International Committee of Medical Journal Editors (ICMJE) criteria

for authorship of this manuscript, take responsibility for the integrity of the work as a whole, and have given final approval for the version to be published.

Access: This article is freely accessible at touchENDOCRINOLOGY.com ( ) Touch Medical Media 2021.

Received: 21 October 2020

Accepted: 5 February 2021

Published Online: 23 March 2021

Citation: touchREVIEWS in Endocrinology. 2021;17(1):2-4

Corresponding Author: John Doupis, Department of Internal Medicine and Diabetes Salamis Naval and Veterans Hospital, Salamis Naval Base, 18900, Salamis, Attiki, Greece. E: john.doupis@harvard-alumni.org

Support: No funding was received for the publication of this article.
Diabetes mellitus is a chronic disease that affects many people around the world. Sedentary lifestyle is considered a main risk factor for type 2 diabetes mellitus (T2DM). As presented in this editorial, the advantages of activity in preventing and treating T2DM are widely recognized. Exercise training improves glycaemic control, body composition, cardiorespiratory wellness, physical functioning and prosperity in patients with T2DM or pre-diabetes. ${ }^{1}$ Lifestyle modification that involves exercise training now represents a main strategy in diabetes prevention. The benefits of exercise prescription in patients with diabetes include the improvement of blood glucose and lipid level control as well as the reduction of body weight and blood pressure. ${ }^{2}$ Furthermore, psychological benefits may follow, such as decreasing anxiety or depression and improvement of sleep quality. In order to prevent diabetes, people often focus on medication and diet control, while the benefits of exercise can overlooked by patients..$^{1{ }^{3}}$ Combined exercise (aerobic and resistance training) should be a key component of any treatment plan for all patients at risk with T2DM.,4

Exercise and diet, in combination with behavioural modification in patients with impaired glucose tolerance, may be superior to medication use in delaying or even preventing the development of T2DM, as shown in the Diabetes Prevention Programme. ${ }^{5,6}$ In this large, multi-centre, randomized, placebo-controlled clinical trial that involved 2,161 high-risk individuals with impaired glucose tolerance, the participants that received intensive counselling on effective diet, exercise and behaviour modification lowered their risk of developing diabetes by $58 \% .{ }^{5}$ In the metformin-treated group the incidence of T2DM was decreased by $31 \%$ for both men and women. ${ }^{6}$ The increased physical activity plus the reduction of daily fat consumption in the diet led to significant weight loss, but only the reduction of body weight was independently associated with decreased progression of impaired glucose tolerance on T2DM. ${ }^{7}$

\section{Effects of exercise on metabolism and the regulation of blood sugar}

Insulin action can be modified depending on the intensity and the frequency of the training programme. ${ }^{8,9}$ After aerobic exercise, muscle glucose uptake remains high during the early postexercise period via insulin-independent mechanisms; insulin sensitivity is markedly reduced and improvement in insulin action may last for 24 hours. ${ }^{8,10,11}$ Contracting muscles during prolonged exercise leads to elevated glucose uptake and contributes to the reduction of hyperglycaemia in the post-absorptive state in patients with T2DM. ${ }^{12}$ Regular aerobic training boosts muscle insulin sensitivity in individuals with pre-diabetes and T2DM in proportion to exercise volume.,10,12 Neither prolonged moderate nor intermittent high intensity exercise performed in the postprandial state alters glucose or insulin response to a subsequent meal. ${ }^{12} \mathrm{~A}$ combination of endurance exercises with resistance training may be of greater benefit, and high intensity interval training may be superior to continuous aerobic training in adults with diabetes.9.12

It is well known that even a single bout of exercise has an explicit effect on the metabolism of people with T2DM, including an increase of insulin-mediated glucose disposal both in the liver 
and the muscle tissue, and the improvement of glucose tolerance. ${ }^{13}$ A brief period of exercise, however, is insufficient to yield the longterm metabolic benefits of repeated training on a daily basis and, as a result, the permanent increase of maximal oxygen consumption, the improvement of body composition and other cardiovascular parameters, and the maintenance of body weight reduction are not guaranteed. ${ }^{14}$

\section{Types of exercise and general recommendations}

Different types of training, including aerobic, resistance and flexibility exercises, could be recommended, but the intensity, duration and frequency of the exercises, as well as some safety measures, should be explained to patients when prescribing a training programme. ${ }^{1,3}$ Aerobic exercise involves repeated and continuous movement of large muscle groups. Activities like walking, cycling, jogging and swimming rely primarily on aerobic energy-producing systems. Resistance (strength) training includes exercises with the use of free weights, weight machines, body weight and resistance bands (multi-joint exercises). . $, 8,15^{-1}$ Flexibility exercises improve range of motion around the joints. Balance exercises benefit gait and prevent falls. Activities like tai chi and yoga combine flexibility, balance and muscle strength improvement at the same time. ${ }^{3,8,11}$

Walking, or other low to moderate intensity aerobic exercises, 2-7 days a week (150 minutes in total) or jogging or other high intensity exercises 3 days a week (90 minutes in total), are generally recommended in order to improve glycaemic control, facilitate weight management and reduce the risk of cardiovascular disease. ${ }^{3.4}$ In order to achieve long-term maintenance of weight loss, 7 hours per week of moderate to vigorous aerobic physical activity may be necessary. ${ }^{16}$ For most patients with diabetes, their physical activity should be distributed over 3-5 days per week with no more than 2 consecutive days without physical activity, in order to attain continuous glycaemic improvement. ${ }^{17}$ Weight lifting or other muscle-strengthening resistance exercises, 3 days a week, could be combined with aerobic training. ${ }^{34,15}$ Given the fact that higher intensity exercise is correlated with increased cardiovascular risk, greater chance for injury and lower compliance compared to lower intensity exercise, people with diabetes who have been inactive should initially engage in physical activity of low to moderate intensity. The intensity of their physical activity should be monitored..$^{14}$ Finally, the mode of exercise should be individualized depending on the patient's characteristics; for example, stationary cycling or swimming would be preferable to slow walking for obese individuals or patients with peripheral neuropathy or microvascular disease. ${ }^{16}$

Sedentary lifestyle has been clearly associated with a doubled risk of all-cause cardiovascular mortality, exceeding, in some cases, other classic risk factors for cardiovascular disease, such as diabetes. ${ }^{18,19}$ Environmental and technological advances, in combination with the impact of urbanization and mechanization on the modern lifestyle contribute significantly to the lack of exercise in the population, despite the recommended guidelines and the proven health benefits of daily physical activity. ${ }^{20}$ The development of behavioural modification techniques in order to improve exercise habits in the general public necessitates clarification of the most favourable type of activity, the amount of time and intensity required and the identification of acceptable and effective exercise derivations. ${ }^{21}$

Given the existing behavioural challenges in improving physical activity, regular physician counselling regarding exercise is recommended in order to limit the deleterious effects of a sedentary lifestyle.22

\section{Pre-exercise health screening precautions}

In most people with diabetes, low intensity activity is generally recommended without any previous testing. ${ }^{8,16}$ Physical activity has some potential health risks for people with diabetes, including acute complications like cardiac events, hypoglycaemia and hyperglycaemia. In low and moderate intensity activity undertaken by adults with T2DM, the risk of exercise-induced adverse events is low. ${ }^{8}$ In individuals with T1DM at any age and those with T2DM who require insulin therapy, the most usual exercise-induced adverse event is hypoglycaemia. ${ }^{23,24}$ Other common problems in these patients may include an acute rise in blood glucose during high intensity, short-term exercise and ketosis as a response to strenuous exercise. According to the American Diabetes Association guidelines, adequate glycaemic control is needed prior to physical activity. Exercise should be avoided if fasting glucose is $>250$ $\mathrm{mg} / \mathrm{dL}$ and blood or urine ketones are elevated; carbohydrates should be ingested if glucose is $<100 \mathrm{mg} / \mathrm{dL}$, and blood glucose monitoring should be carried out before, during and after physical activity; and the consumption of food during extensive exercise is suggested in order to avoid hypoglycaemia. ${ }^{8}$

No current research suggests that any screening protocol beyond usual diabetes care reduces the risk of exercise-induced adverse events in asymptomatic individuals with diabetes. Thus, pre-exercise medical clearance is not necessary for asymptomatic individuals who engage in low to moderate intensity exercises that do not exceed the demands of brisk walking or everyday living and who already receive diabetes care consistent with the current guidelines. ${ }^{8}$ However, in cases where an increase of exercise intensity is desired, patients with diabetes may benefit from a referral to a healthcare provider for a check up and a possible exercise stress test before starting such activities. Effective screening for coronary artery disease, peripheral vascular disease, nephropathy, retinopathy and peripheral and autonomic neuropathy will determine the risk of acute complications and identify the most appropriate physical activities to avoid or to limit. ${ }^{25}$

In individuals with both known and unknown coronary artery disease, exercise may precipitate angina, myocardial infarction, arrhythmia and sudden cardiac death; therefore, a graded exercise stress test before initiating exercise is advised. ${ }^{25}$ In patients with proliferative retinopathy, there are considerable restrictions on the type and intensity of the exercise programme that can be prescribed. The risk of ocular damage, vitreous haemorrhage and retinal detachment remains high during exercises that increase blood pressure and involve Valsalva manoeuvres. As a result, activities that are jarring, traumatic or involve excessive strain (e.g. weight lifting) are contraindicated; swimming, walking or stationary cycling are recommended for these individuals, but regular ophthalmology follow up every $1-2$ months is mandatory. ${ }^{26}$

Autonomic neuropathy reduces cardiac response to exercise and is strongly associated with high cardiovascular disease, so cardiac workup is necessary before initiating exercise. ${ }^{27}$ In individuals with peripheral neuropathy there is a high prevalence of ulcers and charcot foot morphology; therefore, non-weight-bearing exercise is the most appropriate form of training for these patients. ${ }^{25}$ There are no particular limitations to physical activity in patients with diabetic nephropathy; exercise may cause microalbuminuria due to an increase in blood pressure, but there is no evidence that exercise leads to progression of nephropathy. The risk factors for nephropathy are similar to those for coronary artery disease, so appropriate pre-exercise screening should be performed, as mentioned above.28 


\section{Diabetes and exercise during pregnancy}

Despite the physiological changes that occur during pregnancy in response to the development of the foetus and the increased metabolic demands of both mother and foetus, regular physical activity is as beneficial in pregnant women as in non-pregnant individuals. ${ }^{29}$ Maternal benefits include the decreased risk of excessive weight gain, gestational diabetes mellitus and gestational hypertension; reduced incidence of muscle cramps, lower limb oedema and musculoskeletal discomfort; and prevention of urinary incontinence, reduced symptoms of depression and promotion of overall wellness. ${ }^{30}$

Furthermore, exercise during pregnancy is associated with a decreased number of women who required insulin, a shorter labour and a decreased incidence of operative delivery. Foetal health benefits related to the mother's regular physical activity include reduced fat mass, advanced neurobehavioral maturation and improved stress resistance. ${ }^{31}$ According to the most recent guidelines, 60-150 minutes of aerobic exercise per week with an upper limit of 30 minutes/day is generally recommended.
A gradual, weekly increase of physical activity in combination with vigorous exercises and light weight training could be added to the training routine of healthy pregnant women. Among the international guidelines, discrepancies exist regarding the recommended intensity of exercise. ${ }^{31}$ More studies are needed to identify the most effective type and duration of activity and to evaluate the effect of regular training in pregnant women with pre-existing diabetes. ${ }^{32}$

\section{Conclusion}

Exercise plays a cornerstone role in the management of patients with diabetes due to the proven overall health benefits that a proper, regular training routine can offer. The adaptation of regular training for everyday living of these individuals is crucial and can be achieved through effective counselling offered by healthcare providers. The benefits of a training programme should always outweigh any possible risks. The elimination of exercise-induced complications demands proper pre-exercise screening and close monitoring of individuals with high cardiovascular risk. 口
1. Esteghamati A, Hassabi M, Halabchi F, Bagheri M. Exercise prescription in patients with diabetes type 2. J Diabetes Metab Disord. 2008;7:2

2. O'Hagan C, De Vito G, Boreham CAG. Exercise prescription in the treatment of type 2 diabetes mellitus: Current practices, existing guidelines and future directions. Sports Med. 2013;43:39-49.

3. Mendes R, Sousa N, Almeida A, et al. Exercise prescription for patients with type 2 diabetes - A synthesis of international recommendations: Narrative review. Br I Sports Med. 2016;50:1379-81.

4. Hordern MD, Dunstan DW, Prins JB, et al. Exercise prescription for patients with type 2 diabetes and pre-diabetes: A position for patients with type 2 diabetes and pre-diabetes: A post
statement from Exercise and Sport Science Australia. statement from Exercise and Sp

5. Diabetes Prevention Program Research Group; Knowler WC, Fowler SE, Hamman RF, et al. 10-year follow-up of diabetes incidence and weight loss in the Diabetes Prevention Program Outcomes Study. Lancet. 2009;374:1677-86.

6. Knowler WC, Barrett-Connor E, Fowler SE, et al. Reduction in the incidence of type 2 diabetes with lifestyle intervention or metformin. N EngI J Med. 2002;346:393-403.

7. Hamman RF, Wing RR, Edelstein SL, et al. Effect of weight loss with lifestyle intervention on risk of diabetes. Diabetes Care. 2006;29:2102-7.

8. Colberg SR, Sigal RJ, Yardley JE, et al. Physical activity/exercise and diabetes: A position statement of the American Diabetes Association. Diabetes Care. 2016;39:2065-79.

9. Suh SH, Paik IY, Jacobs KA. Regulation of blood glucose homeostasis during prolonged exercise. Mol Cells. 2007;23:272-9.

10. Balducci S, Sacchetti M, Haxhi J, et al. Physical exercise as therapy for type II diabetes. Diabetes Metab Res Rev. 2014;32:13-23.

11. Thomas D, Elliott EJ, Naughton GA. Exercise for type 2 diabetes mellitus. Cochrane Database Syst Rev. 2006;(3):CD002968.

12. Galbo H, Tobin L, Van Loon LC. Responses to acute exercise in type 2 diabetes, with an emphasis on metabolism and interaction with oral hypoglycemic agents and food intake. Appl Physiol Nutr Metab. 2007;32:567-75

13. Devlin JT, Hirshman M, Horton ED, Horton ES. Enhanced peripheral and splanchnic insulin sensitivity in NIDDM men after single bout of exercise. Diabetes. 1987;36:434-9.

14. Pan $B, G e L$, Xun $Y Q$, et al. Exercise training modalities in patients with type 2 diabetes mellitus: A systematic review and network meta-analysis. Int I Behav Nutr Phys Act. 2018:15:1-14.

15. Kirwan JP, Sacks J, Nieuwoudt S. The essential role of exercise in the management of type 2 diabetes. Cleve Clin I Med. in the managem

16. Sigal RJ, Kenny GP, Wasserman DH, Castaneda-Sceppa C Physical activity/exercise and type 2 diabetes. Diabetes Care. 2004;27:2518-39

17. Sigal RJ, Kenny GP, Wasserman DH, et al. Physical activity/ exercise and type 2 diabetes: A consensus statement from the American Diabetes Association. Diabetes Care. 2006;29:1433-8.

18. Blair SN, Kohl HW, Paffenbarger RS, et al. Physical fitness and all-cause mortality: A prospective study of healthy men and women. JAMA. 1989;262:2395-401.

19. Blair SN, Kampert JB, Kohl HW, et al. Influences of cardiorespiratory fitness and other precursors on cardiovascular disease and all-cause mortality in men and women. JAMA. 1996:276:205-10.

20. Battle-Horgen K, Brownell KD. Confronting the Toxic Environment: Environmental and Public Health Actions in a World Crisis. In: Wadden TA \& Stunkard AJ (eds). Handbook of Obesity Treatment, New York: Guilford Press, 2002;95-107.

21. Dunn AL, Anderson RE, Jakicic JM. Lifestyle physical activity interventions: History, short- and long-term effects and recommendations. Am J Prev Med 1998;15:398-412.
22. Preventive Services Task Force. The Guide to Clinical Preventive Services. (AHRQ Publication No. 06-0588), Rockville, MD: Agency for Healthcare Research and Quality, 2006. Available at: www.ncbi.nlm.nih.gov/books/NBK61778/ (accessed 22 March 2021).

23. Chase HP, Dixon B, Pearson J, et al. Reduced hypoglycemic episodes and improved glycemic control in children with type 1 diabetes using insulin glargine and neutral protamine hagedorn insulin. J Pediatr. 2003;143:737-40.

24. MacDonald MJ. Postexercise late-onset hypoglycemia in insulin-dependent diabetic patients. Diabetes Care. 1987;10:584-8.

25. Rydén L, Grant PJ, Anker SD, et al. ESC guidelines on diabetes, pre-diabetes, and cardiovascular diseases developed in collaboration with the EASD. Eur Heart J. 2013;34:3035-87.

26. Tuomilehto J, Lindström J, Eriksson JG, et al. Prevention of type 2 diabetes mellitus by changes in lifestyle among subjects with impaired glucose tolerance. N Eng/ J Med. 2001;344:1343-50.

27. Freeman R. Diabetic autonomic neuropathy. Handb Clin Neurol. 2014;126:63-79

28. Huttunen NP, Kaar M, Puukka R, Akerblom HK. Exercise-induced proteinuria in children and adolescents with type 1 diabetes. Diabetologia. 1981;21:495-7.

29. Melzer K, Schutz Y, Boulvain M, Kayser B. Physical activity and pregnancy: cardiovascular adaptations, recommendations and pregnancy outcomes. Sports Med. 2010;40:493-507.

30. Nascimento SL, Surita FG, Cecatti JG. Physical exercise during pregnancy: a systematic review. Curr Opin Obstet Gynecol. 2012;24:387-94

31. Savvaki D, Taousani E, Goulis DG, et al. Guidelines for exercise during normal pregnancy and gestational diabetes: a review of international recommendations. Hormones (Athens) 2018;17:521-9.

32. Peters TM, Brazeau AS. Exercise in pregnant women with diabetes. Curr Diab Rep. 2019;19:80 